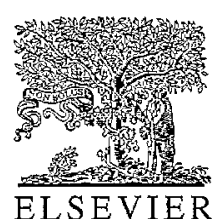

Materials Science and Engineering B42 (1996) 38-42

MARTAL

SelEMEE

EMGHEERTN

\title{
Cathodoluminescence microscopy of doped GaSb crystals
}

\author{
B. Ménder $z^{a, *}$ J. Piquerasa ${ }^{a}$ P.S. Dutta ${ }^{b}$, E. Dieguez ${ }^{b}$ \\ a Departamento de Física de Materiales, Facultad de Fisicas, Universidad Complutense, E-28040 Madrid, Spain \\ ${ }^{-}$Departamento de Física de Materiales, Universidad Autónoma, Cantoblanco, 28049 Madrit, Spain
}

\begin{abstract}
We present the homogeneity and luminescence properties of bulk GaSb obtained by the cathodoluminescence (CL) technique in the scanning electron microscope. The samples used in this study are as-grown undoped and impurity diffused (tellurium) and doped (chromium) material. CL investigations have revealed a non uniform distribution of native defects in GaSb wafers. Post growth annealing in vacuum, gallium or antimony atmospheres causes an increase in homogeneity in CL images. Te diffusion and $\mathrm{Cr}$ doping provides new information about defects in GaSb. CL images and CL spectra recorded in these samples support that the type of defects formed is a function of diffusion time and impurity concentration.
\end{abstract}

Keywords: Cathodoluminescence; Diffusion time; Tellurium

\section{Introduction}

In recent years, gallium antimonide has generated interest as a potential substrate material for various lattice-matched devices operating in the range of about $0.3-1.6 \mathrm{eV}$. However, significant progress has to be made both in material characterization and processing before it can be widely employed for device applications. There are several reports on the growth of bulk GaSb single crystals [1-3] but little information is available regarding the characterization of defects in GaSb crystals. It is well known that the quality of epitaxial layers and the behaviour of devices made from them depend on the structural quality of the substrate. For this reason characterization of defects in bulk $\mathrm{GaSb}$ is a necessary step for the practical application of this material for micro and optoelectronic devices. The interest of defect studies refers to undoped and to crystals after doping with different electrically active impurities.

For defect characterization, cathodoluminescence (CL) in the scanning electron microscope (SEM) has been extensively applied to many semiconductors and semiconductor heterostructures. This technique which provides information on the nature and spatial distribution of the defects has been to our knowledge, only

\footnotetext{
* Corresponding author.
}

recently applied to characterize GaSb crystals as reported in previous works of our laboratory $[4,5]$.

Undoped $\mathrm{GaSb}$ is p-type in nature with a high acceptor concentration. Acceptors are intrinsic and are due to gallium vacancies $\left(\mathrm{V}_{\mathrm{G}}\right)$ and gallium antisites $\left(\mathrm{Ga}_{\mathrm{Sb}}\right)$. We have investigated samples after annealing in vacuum, gallium and antimonide atmospheres to examine the evolution and nature of the native acceptors. Due to the presence of high concentration of acceptors impurity doping leads to compensation. In fact, controlled doping to low levels poses problems. One means of achieving that is dopant incorporation by impurity diffusion, which has been given limited attention to date. Te impurity can be used to obtain n-type material in a thin layer below the surface. In this paper we present results concerning the nature of luminescent centres after $\mathrm{Te}$ diffusion. During the course of this investigation on doped GaSb we have also carried out chromium doping. Chromium is known to create deep levels in III$\mathrm{V}$ compounds like GaAs causing a semiinsulating behaviour. To our knowledge there are no reports on semiinsulating bulk GaSb. To see the possible effect of $\mathrm{Cr}$ doping on the native defect concentration, single crystals of $\mathrm{GaSb}: \mathrm{Cr}$ were grown by the horizontal Bridgman technique and investigated by $\mathrm{CL}$. In the present work, CL in the SEM is used to study the nature of defects in undoped as well as in $\mathrm{Te}$ and $\mathrm{Cr}$ doped samples. 


\section{Experimental method}

The samples used in this work were $\mathrm{GaSb}$ and $\mathrm{GaSb}: \mathrm{Cr}$ single crystals grown by the vertical and horizontal Bridgman method. For the annealing treatments the wafers were prepared by conventional chemo-mechanical polishing. For the gallium annealing, $6 \mathrm{~N}$ pure gallium was spread on the wafer surface at room temperature. For annealing in antimony atmosphere, $6 \mathrm{~N}$ pure antimony balls were kept along with the wafer, Other wafers were annealed under a vacuum of $10^{-\sigma}$ Torr. The annealing temperature and time was kept constant at $500^{\circ} \mathrm{C}$ and $12 \mathrm{~h}$, respectively, for all the treatments. The Te-diffusion experiments were carried out in evacuated and sealed quartz ampoules at $500^{\circ} \mathrm{C}$ for 2, 15 and $24 \mathrm{~h}$. Details of the diffusion experiments will be provided elsewhere [6].

For CL measurements a Hitachi S-2500 scanning electron microscope was used. Samples were at $77 \mathrm{~K}$ and the luminescence signal was detected with a North Coast EO-817 germanium detector.

\section{Results and discussion}

CL spectra of as-grown undoped samples (Fig. 1) show that two main emission bands peaked at about 796 and $775 \mathrm{meV}$, respectively, and a weaker emission at $756 \mathrm{meV}$. The $796 \mathrm{meV}$ band corresponds to the band-band transition and the $775 \mathrm{meV}$, usually known as band $\mathrm{A}$, has been attributed $[7,8]$ to the presence of the acceptor level $\mathrm{V}_{\mathrm{Ga}}-\mathrm{Ga}_{\mathrm{Sb}}$. The band at $756 \mathrm{meV}$

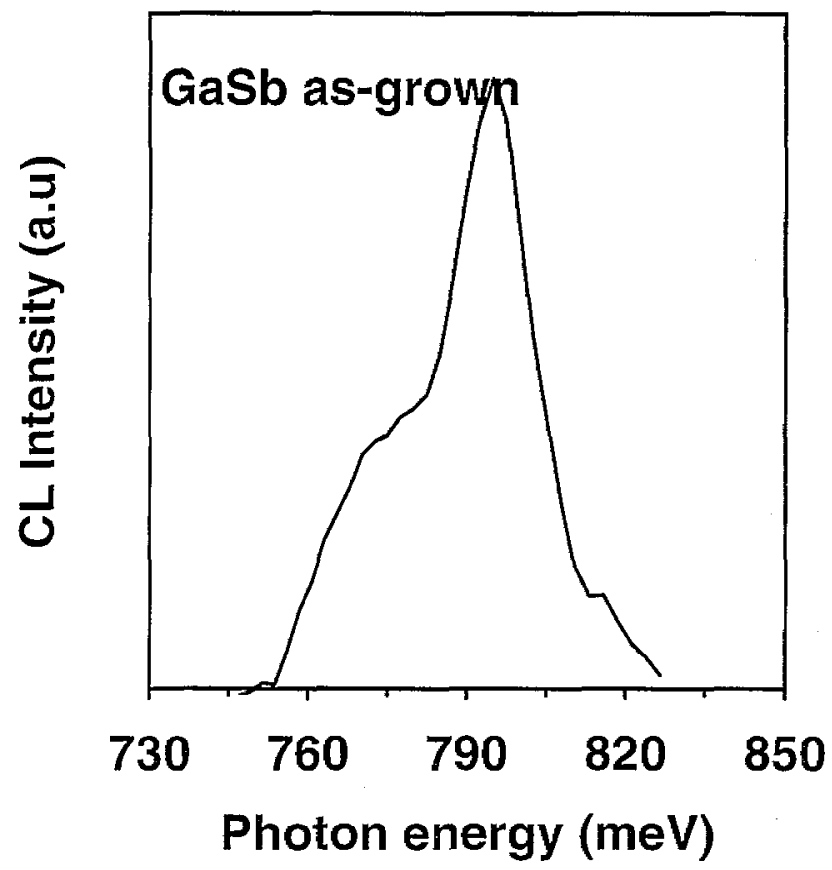

Fig. 1. CL spectrum at $77 \mathrm{~K}$ of a sample of as-grown undoped GaSb.
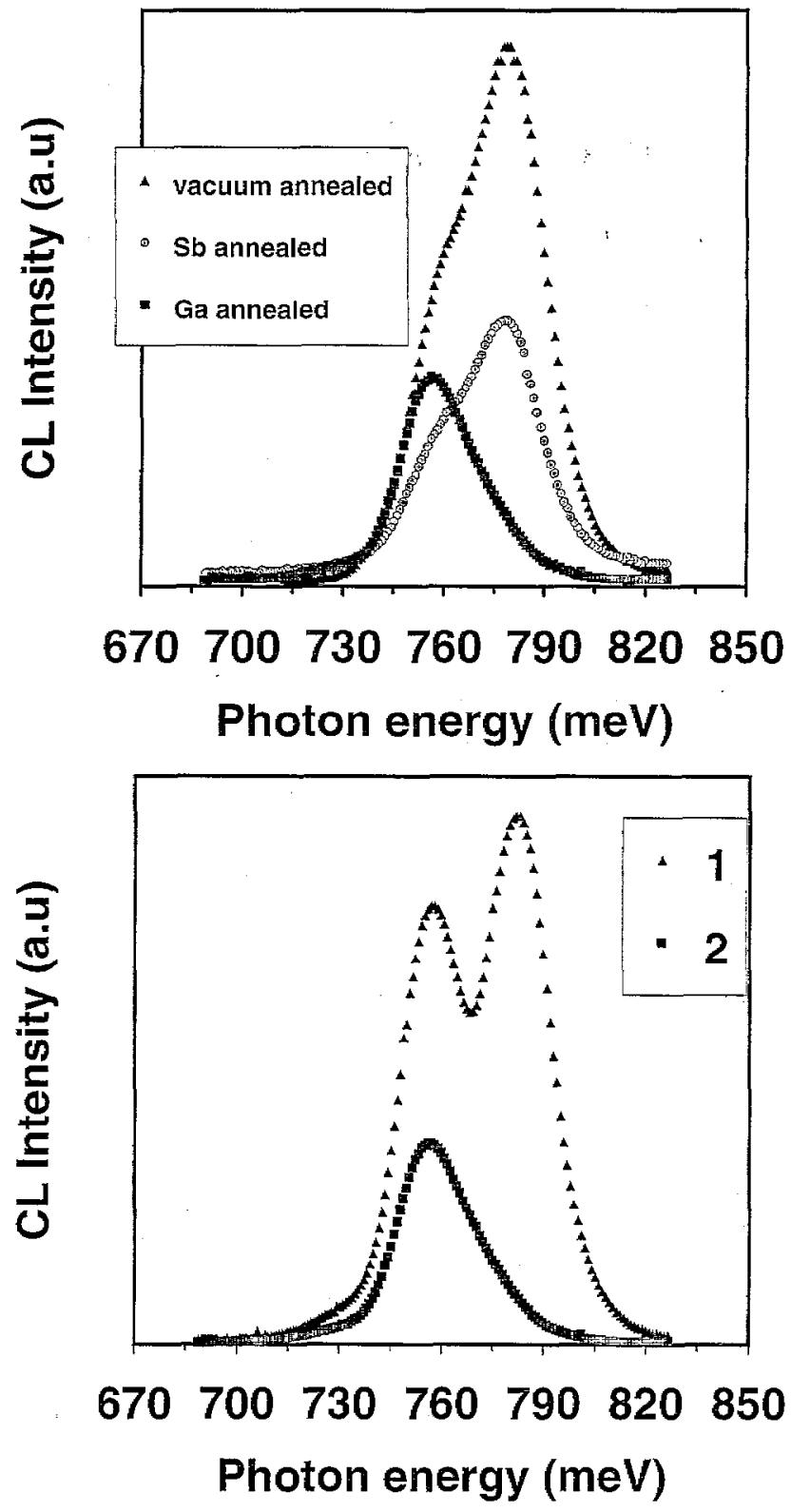

Fig. 2. CL spectra at $77 \mathrm{~K}$ of (a) vacuum, antimony and gallium annealed $\mathrm{GaSb}$ at $500^{\circ} \mathrm{C}, 12 \mathrm{~h}$, and (b) $\mathrm{CL}$ spectra from gallium anmealed obtained from different positions in the sample.

(designated as band $\mathrm{B}$ ) has been related to a complex $\left(\mathrm{V}_{\mathrm{Ga}}-\mathrm{Ga}_{\mathrm{Sb}}-\mathrm{V}_{\mathrm{Ga}}\right)$ arising from an excess of $\mathrm{Ga}$ vacancies [9], but its origin is not yet well determined. Annealing treatments provide some information on the possible origin of the luminescence bands (Fig. 2). For comparison, vacuum, $\mathrm{Sb}$ and Ga-annealed samples have been studied. CL spectra of annealed samples (Fig. 2(a)) reveal only the 775 and $756 \mathrm{meV}$ bands. Fig. 2(b) shows CL spectra of the Ga annealed sample taken from two different positions in the sample, revealing that the intensity of the $756 \mathrm{meV}$ transition enhances drastically (Fig. 2(b)). This result suggests that the band $\mathrm{B}$ is associated with a defect involving excess of gallium as a gallium antisite or related complex. 
The CL images of as grown crystals show contrast related to subboundaries (Fig. 3(a)). Similar images are obtained in vacuum annealed samples but after annealing in $\mathrm{Sb}$ dark precipitate defects are observed (Fig. 3(b)). The $\mathrm{Ga}$ annealed sample shows a rather homogeneous distribution of $\mathrm{CL}$ intensity, which indicates the possibility of using $\mathrm{Ga}$ annealing treatments as a procedure leading to wafer homogeneity.

In Te-diffused samples the features observed in CL images are a function of the diffusion time. After short

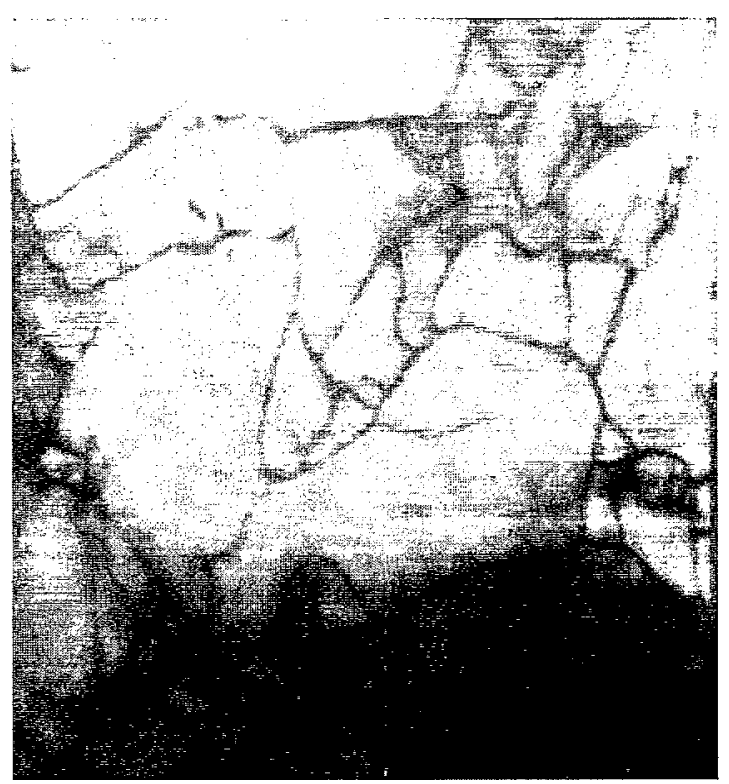

(a)

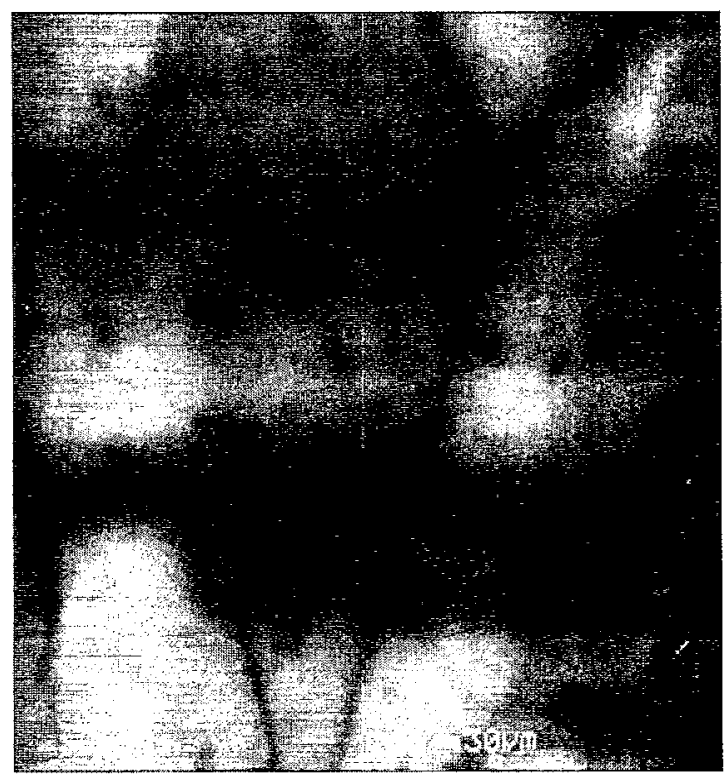

(b)

Fig. 3. CL images of (a) as-grown and (b) gallium annealed samples.

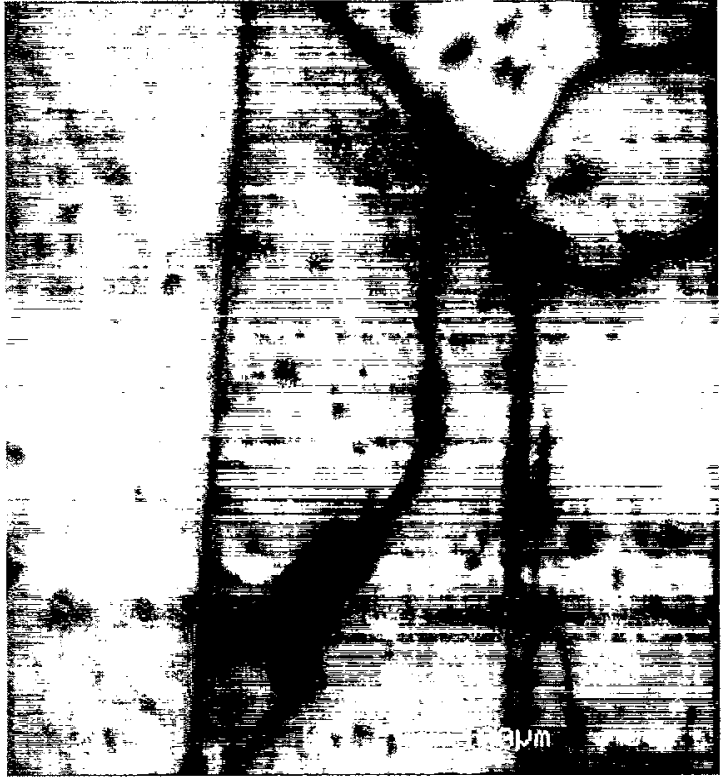

Fig. 4. CL image of Te-diffused GaSb (24 h),

diffusion time $(2 \mathrm{~h})$ the contrast is similar to that of the undoped samples but show, as an additional feature, small dark dots probably due to decorated dislocations. Increasing annealing time leads to the formation of precipitate defects (Fig. 4) which could either be Te precipitates or the $\mathrm{Ga}_{2} \mathrm{Te}_{3}$ precipitates observed by transmission electron microscopy by other authors [10]. CL spectra of Te-diffused samples (Fig. 5) show a peak at $744 \mathrm{meV}$ which has been attributed [11] to transition from conduction band to an acceptor state of the complex $\mathrm{V}_{\mathrm{Ga}}-\mathrm{Ga}_{\mathrm{Sb}}-\mathrm{Te}_{\mathrm{Sb}}$. The spatial distribution of this defect seems to be related to the presence of dislocations as CL images show. With an increase in $\mathrm{Te}$ concentration, the intensity of $744 \mathrm{meV}$ peak increases (Fig. 5(a)), but in the sample with highest Te concentration, the $744 \mathrm{meV}$ almost disappears while the $756 \mathrm{meV}$ becomes the dominant transition (Fig. 5(b)). This effect can be explained by increasing volatization of $\mathrm{Sb}$ after long diffusion times. This results in $\mathrm{Ga}$ excess and the effect on CL spectra is similar to that caused by an excess described in the $\mathrm{Ga}$-annealed undoped samples.

The effect of $\mathrm{Cr}$ on the structure of luminescent centres, was studied on an ingot of approximately 20 $\mathrm{mm}$ length. $\mathrm{CL}$ spectra were recorded along the length and the influence of $\mathrm{Cr}$ concentration on the emission bands could be investigated. Even though there are no data, from electrical resistivity measurements, on the segregation coefficient of $\mathrm{Cr}$ in $\mathrm{GaSb}$, we have observed that along the growth direction there is a segregation of Cr. Fig. 6 shows the CL spectra corresponding to $\mathrm{Cr}$-rich and Cr-poor ends of the ingot. The spectra have been deconvoluted and the best fits obtained. In regions with a low concentration of $\mathrm{Cr}$ (Fig. 6(a)), a broad 
peak around $770 \mathrm{meV}$ appears. This peak can be decomposed into two peaks at 778 and $770 \mathrm{meV}$ of comparable intensity. In the opposite side of the ingot, the broad band corresponds to the 778 and $756 \mathrm{meV}$ bands (Fig. 6(b)). In comparison with undoped crystals, all spectra obtained from the $\mathrm{Cr}$ doped ingot show a reduced intensity of band $A$ which means that $\mathrm{Cr}$ doping decreases the concentration of the native acceptor responsible for this emission. The $\mathrm{CL}$ observations in $\mathrm{GaSb}: \mathrm{Cr}$ can be explained by the formation of complexes involving $\mathrm{Cr}$ during the growith of the doped crystals. The formation of native defects in $\mathrm{GaSb}$ is
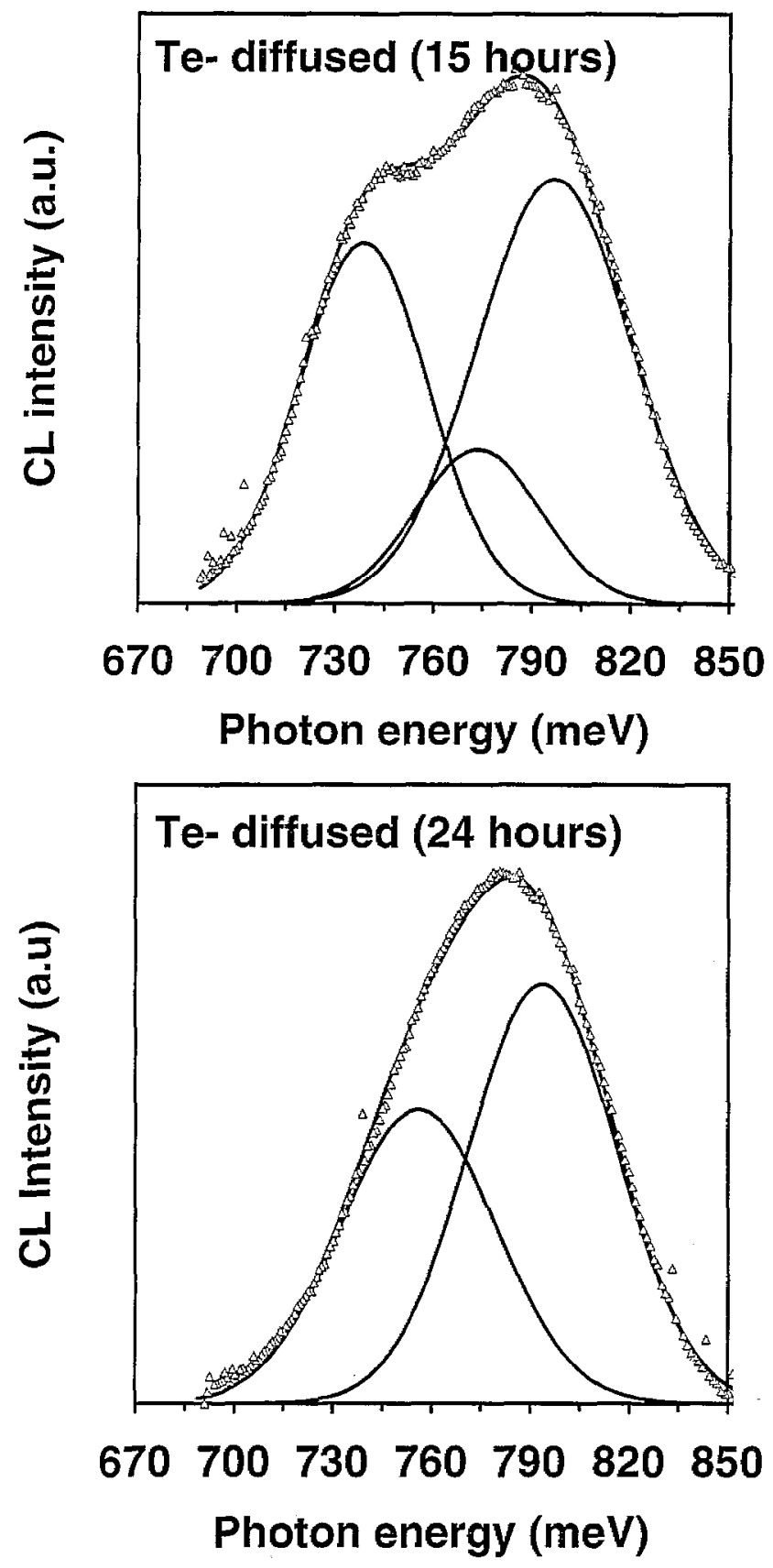

Fig. 5. CL spectra of Te-diffused samples (a) $15 \mathrm{~h}$ and (b) $24 \mathrm{~h}$.
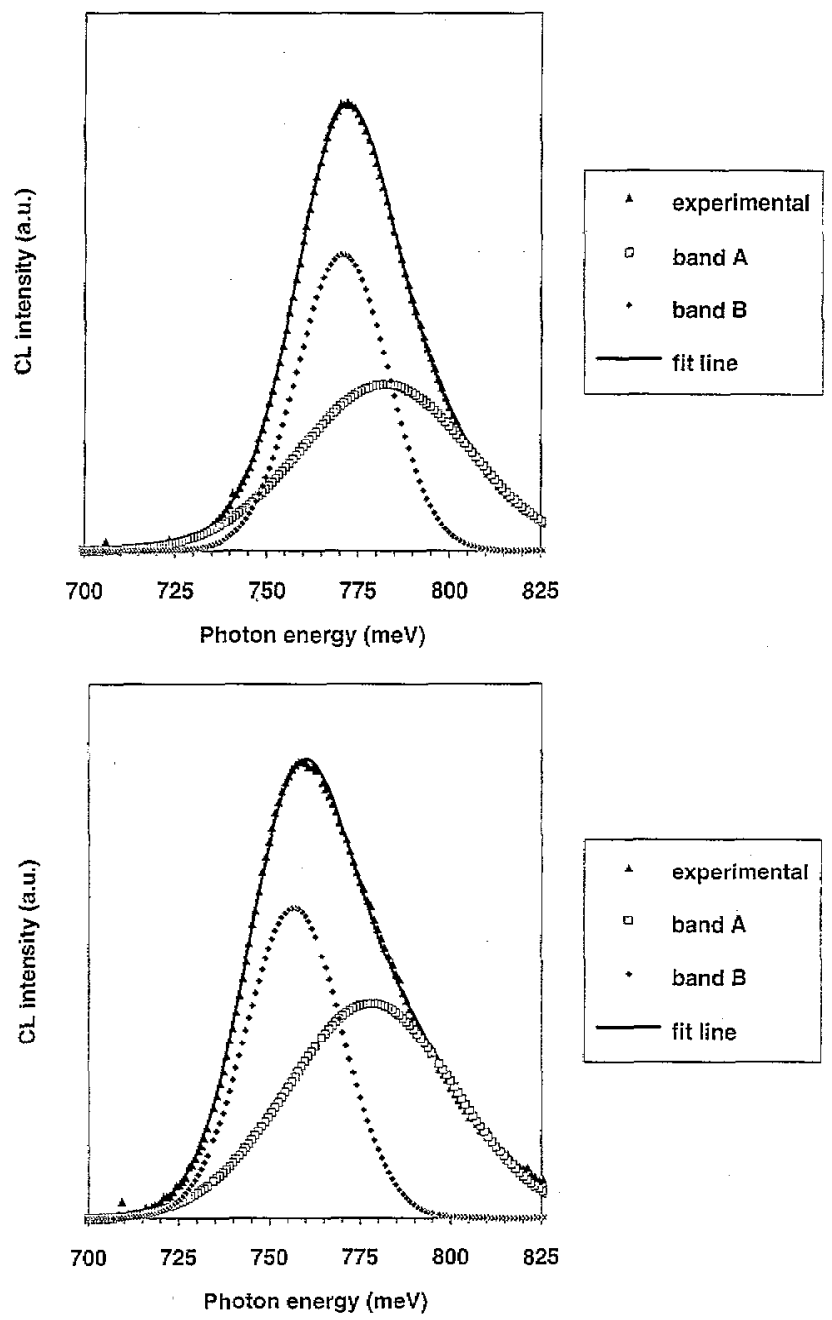

Fig. 6. CL spectra of $\mathrm{Cr}$ doped GaSb (a) low $\mathrm{Cr}$ and (b) high $\mathrm{Cr}$ concentration.

believed to be initiated by the volatilization of $\mathrm{Sb}$ with the formation of $\mathrm{Sb}$ vacancies. This leads in a further step to the formation of Ga-antisites with the schema $\mathrm{Ga}_{\mathrm{Ga}}+\mathrm{V}_{\mathrm{Sb}} \rightarrow \mathrm{Ga}_{\mathrm{Sb}}+\mathrm{V}_{\mathrm{Ga}}$. In the presence of $\mathrm{Cr}$, however, the complex $\mathrm{Cr}-\mathrm{V}_{\mathrm{Sb}}$ is formed as observed in the case of GaSb:Mn by photoluminescence and electron spin resonance [12]. This results in an excess of $\mathrm{Ga}$ in the sample leading to an increase in the $756 \mathrm{meV}$ band which, as described above, increases in $\mathrm{Ga}$ annealed samples.

\section{Conclusions}

In conclusion, we have analyzed the nature and distribution of defects in GaSb by CL in the SEM through the evolution of luminescence bands. The samples investigated were as grown, Te diffused and $\mathrm{Cr}$ doped $\mathrm{GaSb}$. The two main emission bands related to native defects are band $A(777 \mathrm{meV})$ and band $B(756$ $\mathrm{meV})$. The origin of the first one scems to be clcar but 
the nature of centres that induce band $B$ remains unknown. In a previous work made on annealed undoped samples we have suggested that $\mathrm{Ga}$ atoms are involved in the center responsible for the $\mathrm{B}$ emission. In this work, we have obtained new results concerning the origin of band $\mathrm{B}$ when we study $\mathrm{Te}$ diffuse and $\mathrm{Cr}$ doped samples. The results confirm the assumption that an excess of Gallium enhances the $756 \mathrm{meV}$ emission.

\section{References}

[1] C. Woelk and K.W. Benz, J. Cryst. Growth, 27 (1977) 177.

[2] E.T.R. Chidley, S.K. Haywood, A.B. Henriques, N.J. Mason, R.J. Nicholas and P.J. Walker, Semicond. Sci. Technol, 6 (1991) 45 .
[3] T.M. Rossi, D.A. Collins, D.H. Chow and T.C. McGill, Appl. Pllys. Lett., 57 (1990) 2256.

[4] B. Mendez, P.S. Dutta, J. Piqueras and E. Dieguez, Appl. Plys. Lett., 67 (1995) 2648.

[5] G.N. Panin, P.S. Dutta, J. Piqueras and E. Dieguez, Appl. Phys. Lett., 67 (1995) 3584.

[6] P.S. Dutta, B. Mendez, J. Piqueras, E. Dieguez and H.L, Bhat, J. Appl. Phys. H, 80 (1996) 1112.

[7] W. Jakowetz, W. Ruhle, K. Breuninger and M. Pilkuhn, Phys. Stat. Sol.(a), 12 (1972) 169.

[8] M.C. Wu and C.C. Chen, J. Appl. Phys., 72 (1992) 4275.

[9] D. Weler and H. Mehrer, Philos. Mag., A49 (1984) 309.

[10] J. Doerschel and U. Geissler, J. Cryst. Growth, 121 (1992) 781.

[11] A.I. Lebedev and I.A. Strel'nikova, Sov. Phys. Semicond., 13 (1979) 229.

[12] E.I. Georgitse, L.M. Gatsalak, V.I. Ivanov-Omskii, V.F. Masterov, V.A. Smimov and K.F. Shtel'makh, Sov. Phys. Semicon., 26 (1992) 50. 